\title{
Avaliação de Desempenho de Redes de Pesquisa: o Caso do Instituto Nacional de Ciência, Inovação e Tecnologia em Saúde - INCT- Citecs*
}

\section{Evaluation of Research Network Performance: the Case of the National Institute of Science, Innovation and Technology in Health - INCT - Citecs}

\author{
Fabiana Raynal Floriano* * \\ Marcelle de Oliveira Cardoso*** \\ Luis Eugenio Portela Fernandes de Souza**** \\ Rosana Aquino Guimarães Pereira***** \\ Maurício Lima Barreto****** \\ Sebastião Antônio Loureiro de Souza e Silva* ***** *
}

Resumo: As redes de colaboração científica são formas de organização que potencializam açõesde pesquisa, a partir da articulação de diferentes competências. Este estudo objetivou avaliar o desempenho do Instituto Nacional de Ciência, Inovação e Tecnologia em Saúde (Citecs), em 2009 e 2010, através de uma matriz composta por 21 indicadores agrupados em quatro eixos: (1) consolidação da rede, (2) transferência de conhecimento, (3) pesquisa e (4) formação de pessoal. Os resultados revelaram que o Citecs tem atendido aos objetivos propostos, ampliando suas ações nos quatro eixos. Neste período avaliado, destaca-se o aumento

*Este estudo recebeu apoio financeiro do Programa Institutos Nacionais de Ciência e Tecnologia do CNPq, (MCT-CNPq) - Processo n $^{\circ} 57386 / 2008-9$.

**Pesquisadora do Instituto Nacional de Ciência Inovação e Tecnologia em Saúde (INCT-Citecs). Bolsista pós-doc SUS pela Capes. E-mail: fabianaraynal@hotmail.com.

* **:Pesquisadora do Instituto Nacional de Ciência Inovação e Tecnologia em Saúde (INCT-Citecs). Bolsista DTI-II pela CNPq. E-mail: cardosomarcelle@gmail.com.

****Professor adjunto do Instituto de Saúde Coletiva da Universidade Federal da Bahia (ISC/UFBA). Coordenador do Programa Integrado de Economia, Tecnologia e Inovação em Saúde (PECS/ISC/ UFBA). Membro do Comitê Gestor do Instituto Nacional de Ciência Inovação e Tecnologia em Saúde (INCT-Citecs).E-mail: luiseugenio@ufba.br.

*****Professora da Residência de Medicina Social do Instituto de Saúde Coletiva da Universidade Federal da Bahia (ISC/UFBA). Pesquisadora do Instituto Nacional de Ciência Inovação e Tecnologia em Saúde (INCT-Citecs). E-mail: aquino@ufba.br.

****** Professor titular do Instituto de Saúde Coletiva da Universidade Federal da Bahia (ISC/UFBA). Pesquisador e Bolsista de Produtividade I-A do CNPq. Coordenador do Instituto Nacional de Ciência Inovação e Tecnologia em Saúde (INCT-Citecs).E-mail: mauricio@ufba.br.

******Professor emérito do Instituto de Saúde Coletiva da Universidade Federal da Bahia (ISC/UFBA). Bolsista DTI-I do CNPq. Vice-coordenador do Instituto Nacional de Ciência Inovação e Tecnologia em Saúde (INCT-Citecs).E-mail: loureiro@ufba.br. 
dos seguintes indicadores: publicações científicas em conjunto, apoio à formulação de políticas na área da saúde, atividades de educação científica e orientação de alunos de graduação em atividades de iniciação científica. O método utilizado nesta avaliação pode ser adaptado para outras redes de pesquisa com objetivos similares.

Palavras-chave: Avaliação de desempenho. Indicadores de ciência. Tecnologia e inovação. Redes de informação de ciência e tecnologia.

Abstract: Research networks are an organizational form which enhance actions, articulating different competencies. This study aimed to evaluate the overall performance of the National Institute of Science, Innovation and Technology in Health (Citecs) in 2009 and 2010. For the analysis, a matrix was created. It wascomposed of 21 indicators grouped into four components: (1) Strengthening of the network, (2) Knowledge transfer, (3) Research, (4) Training. The results showed that Citecs achieved its goals, increased its activities in the four components.In this period,the following indicators were highlighted, due to their significant rise: peer reviewed papers in collaboration;scientific support tohealthpolicies; science education activities, and undergraduate student in science program supervision. The methods used in this evaluation may be adapted to assess other research networks with similar objectives.

Keywords:Performance evaluation. Science, technology and innovationindicators. Science and technology information networks.

JEL Classification:I23; O32; O38.

\section{1 lntrodução}

Na última década, o Brasil tem buscado avançar na consolidação de um Sistema Nacional de Ciência, Tecnologia e Inovação definindo, dentre as prioridades da política setorial, a construção do marco legal-regulatório, o fortalecimento de atividades de pesquisa e inovação em áreas específicas, bem como a difusão de tecnologias para a inclusão e o desenvolvimento social.

Nesse contexto, foi criado o Programa dos Institutos Nacionais de Ciência e Tecnologia (INCT), no âmbito do Ministério de Ciência e Tecnologia, como instrumento de apoio ao desenvolvimento da Ciência e Tecnologia no país. Atualmente, estão em funcionamento 123 institutos, sendo que novos estão sendo criados nas mais diversas áreas do conhecimento (BRASIL, 2009).

Os INCT são organizados para mobilizar e agregar, com atuação em rede, grupos de pesquisa para desenvolverem pesquisa, formação de recursos humanos e transferência do conhecimento para a sociedade, empresas e governo. A forma 
organizacional em rede, proposta para os Institutos, é tida como eficiente, por permitir melhor aproveitamento dos recursos disponíveis, compartilhamento de informações e articulação de diferentes competências (GRIFFITHS et al., 2000).

Podolny e Page (1998) definem os modelos organizacionais em rede como um conjunto formado por dois ou mais atores que estabelecem trocas entre si, sem que haja uma autoridade externa para arbitrar e resolver eventuais disputas entre os membros. As vantagens de pertencer a uma rede, para esses autores, são: aumentos do aprendizado, da legitimidade e do status científico, benefícios econômicos, eficiência na gestão de recursos e maior autonomia.

A noção de rede tem sido amplamente utilizada, em diversas disciplinas científicas, para descrever o funcionamento de sistemas complexos presentes na natureza e criados pela ação humana (ALMEIDA FILHO, 2005). Este autor define rede como um conjunto de pontos - ou nós - distintos, conectados entre si, de forma que mudanças em qualquer um deles têm ressonância na rede como um todo. Moraes (2004) chama a atenção para o fato de que a noção de rede pressupõe a ideia de movimento, fluxos, alianças e vínculos que não são previsíveis e estáveis, mas, ao contrário, estão em permanente redefinição.

Segundo Barabási et al. (2002), a comunidade científica é um exemplo de sistema complexo organizado em rede, visto que é caracterizada, por natureza, pela interconectividade. A cooperação no processo de construção do conhecimento científico vem sendo incentivada por órgãos financiadores em todo o mundo, através do estímulo à formação e à consolidação de redes formais de pesquisa com o objetivo de potencializar e estimular a colaboração entre grupos de pesquisa distintos.

Devido aos significativos investimentos governamentais brasileiros para implantar os INCT, a avaliação do desempenho dessas redes de pesquisa é de grande importância. Apesar de terem sido desenvolvidas diversas propostas de avaliação do desempenho de organizações, o desempenho de redes é algo pouco explorado e conhecido (WEGNER; RIBEIRO, 2011). Ainda mais escassos são os estudos que propõem metodologias de avaliação do desempenho de redes de pesquisa.

O presente estudo realizou uma avaliação de desempenho tomando como unidade de análise um dos INCT em atuação no Brasil: o Instituto de Ciência, Inovação e Tecnologia em Saúde (Citecs). O Citecs é composto por 13 grupos de pesquisadores vinculados às seguintes instituições: Universidade Federal da Bahia, Instituto Federal de Educação, Ciência e Tecnologia da Bahia e Hospital São Rafael. Criado em 2009, tem por objetivo contribuir para a formulação de políticas de saúde, ao desenvolver tecnologias, pesquisas nas áreas de avaliação econômica e tecnológica em saúde e estudos sobre a dinâmica da inovação. Para avaliar o desempenho do Citecs, este estudo desenvolveu uma matriz avaliativa específica. 


\section{Metodologia}

Realizou-se um estudo descritivo, com base em dados primários, obtidos através de entrevistas com os líderes de 13 grupos de pesquisa que compõem a rede Citecs, e dados secundários, coletados dos currículos Lattes desses pesquisadores.

A matriz avaliativa foi elaborada, utilizando como referencial os critérios de avaliação adotados pelo CNPq para os INCT (BRASIL, 2008): a produção e a transferência do conhecimento, a formação de pessoal e o fortalecimento da rede. A avaliação realizada teve como foco os processos desenvolvidos pela rede e seus resultados, considerando-se as atividades desenvolvidas pelo Citecs e os produtos (outputs) decorrentes das atividades. Dessa forma, a matriz (Quadro 1) foi composta por 21 indicadores agrupados em quatro eixos, de acordo com os seguintes objetivos:

a) Eixo 1: Consolidação da rede - dinâmica da rede, conexão, relação e mecanismos de interação existentes entre os grupos;

b) Eixo 2: Transferência de conhecimento - ações de disseminação e/ou intercâmbio de conhecimento com o governo, empresas e sociedade (UNITED KINGDOM, 2011);

c) Eixo 3: Pesquisa - produção científica medida pelo número de publicações de artigos ou livros e projetos em andamento; $\mathrm{e}$

d) Eixo 4: Formação de pessoal - potencial da rede em contribuir com a Pesquisa $\mathcal{Z}$ Desenvolvimento (PED) em saúde, através da formação de novos pesquisadores.

Por considerar que os eixos possuem igual importância na avaliação de desempenho da rede de pesquisa, não foi atribuído valoração relativa entre eixos, assim como entre indicadores.

A partir dos indicadores da matriz foi construída uma representação gráfica da interação entre os grupos de pesquisa que compõem o Citecs, representados pelos coordenadores. As conexões entre os grupos foram determinadas pelos artigos publicados em parceria, assim como o desenvolvimento de pesquisas em conjunto durante o período avaliado. As conexões potenciais foram definidas através da identificação de temas comuns de pesquisa e da possibilidade de realização de projeto de pesquisa em parceria, relatadas pelos coordenadores durante as entrevistas.

A avaliação do desempenho do Citecs considerou os anos de 2009 e 2010, através da comparação das frequências absolutas dos indicadores que compõem a matriz. O banco de dados foi construído no programa estatístico Epilnfo 6.03 (DEAN, 1998) e as análises descritivas no SPSS 13.0 (SPSS, 2006). 
Quadro 1 - Matriz avaliativa: eixos e indicadores

\begin{tabular}{|c|c|}
\hline Eixos & Indicadores \\
\hline \multirow{4}{*}{ 1. Consolidação da rede } & 1. Publicações em colaboração \\
\hline & 2. Atividades (sessões, workshops, etc) científicas em colaboração \\
\hline & 3. Intercâmbio de alunos e/ou pesquisadores \\
\hline & $\begin{array}{l}\text { 4. Desenvolvimento ou fortalecimento de áreas comuns de experti- } \\
\text { se/interesse dos grupos de pesquisa }\end{array}$ \\
\hline \multirow{7}{*}{$\begin{array}{l}\text { 2. Transferência de } \\
\text { conhecimento }\end{array}$} & 5. Participação em comitês de PËD \\
\hline & $\begin{array}{l}\text { 6. Subsídio à formulação de políticas ou à elaboração de material } \\
\text { técnico }\end{array}$ \\
\hline & 7. Parcerias com governo ou empresas \\
\hline & 8. Atividades de educação científica (para a sociedade, em geral) \\
\hline & 9. Atividades de divulgação (jornais/TV/rádio) \\
\hline & 10. Devolução de resultados para sujeitos da pesquisa \\
\hline & 11. Depósito de patentes \\
\hline \multirow{4}{*}{ 3. Pesquisa } & 12. Publicação de artigos científicos \\
\hline & 13. Publicação de livros \\
\hline & 14. Publicação de capítulos de livro \\
\hline & 15. Projetos de pesquisa em andamento \\
\hline \multirow{6}{*}{ 4. Formação de pessoal } & 16. Orientação de alunos de pós-doutorado \\
\hline & 17. Orientação de alunos de doutorado \\
\hline & 18. Orientação de alunos de mestrado (acadêmico/profissional) \\
\hline & 19. Orientação de alunos de especialização \\
\hline & 20. Orientação de alunos de iniciação científica \\
\hline & 21. Cursos (especialização;disciplina especial; extensão) \\
\hline
\end{tabular}

Fonte: Elaboração própria.

\section{Resultados}

Os indicadores do eixo de consolidação da rede mostraram que os pesquisadores do Citecs publicaram 12 artigos científicos em colaboração no ano de 2009 e, 32 em 2010, realizaram 11 atividades científicas (sessões, workshops, etc.) em colaboração em cada ano e promoveram o intercâmbio de nove alunos e/ou pesquisadores em 2009 e seis em 2010. Ainda no eixo 1, foram identificadas nove conexões já estabelecidas entre os grupos que compõem o Citecs, representados por seus coordenadores, e outras nove conexões potenciais, considerando-se as áreas de pesquisa de interesse comum (Figura 1). 
Figura 1 - Rede de conexões, existentes ${ }^{1}$ e potenciais ${ }^{2}$, entre os coordenadores dos grupos de pesquisa do CITECS, 2010

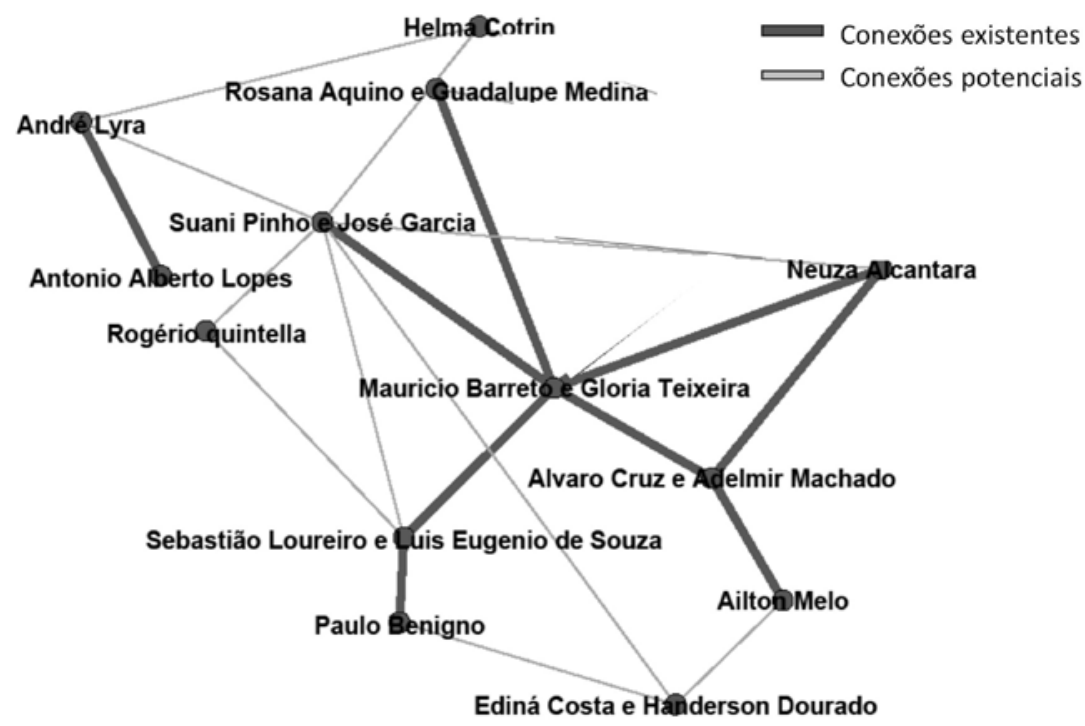

Fonte: Elaboração própria.

Notas: 1. Conexões existentes - artigos publicados em parceria e desenvolvimento de pesquisas em conjunto; 2 . Conexões potenciais - áreas de pesquisa de interesse comum.

Quanto aos indicadores do eixo das atividades de transferência do conhecimento, observou-se que, em 2009, 38 pesquisadores do Citecs participavam em comitês de PED, nove colaboraram com a formulação de políticas ou a elaboração de material técnico e 14 tiveram atividades divulgadas nos meios de comunicação, enquanto, em 2010, esses valores foram 41, 18 e 16, respectivamente.

Os pesquisadores do Citecs desenvolveram dez atividades em parceria com governos ou empresas, em cada ano do estudo, e conduziram duas pesquisas, em 2009 , e sete, em 2010, cujos resultados foram devolvidos para os participantes. Ainda no eixo 2, identificou-se a realização de sete atividades de educação científica, em 2010. Nenhuma patente foi depositada (Figura 2). 
Figura 2 - Distribuição dos indicadores de transferência de conhecimento, CITECS, 2009 e 2010.

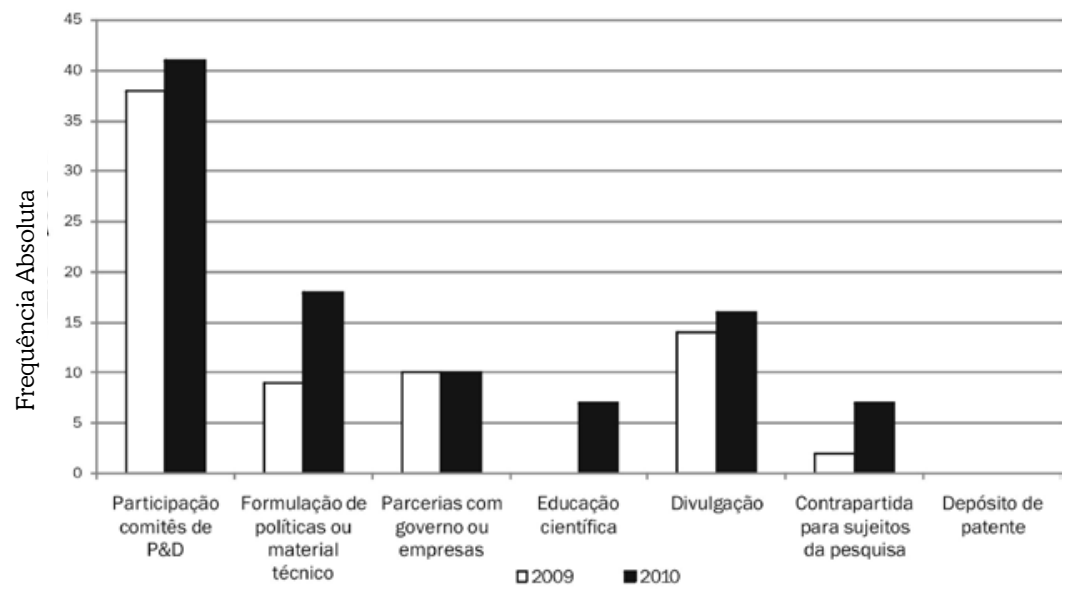

Fonte: Elaboração própria.

Os indicadores do eixo de pesquisa mostraram que o Citecs, em 2009 e 2010, publicou 112 e 145 artigos científicos, três e dois livros e 19 e 20 capítulos de livro, respectivamente. $\mathrm{O}$ número de projetos de pesquisa em andamento foi de $77 \mathrm{em}$ 2009 e 78 em 2010 (Figura 3).

Figura 3 - Distribuição dos indicadores de pesquisa, CITECS, 2009 e 2010.

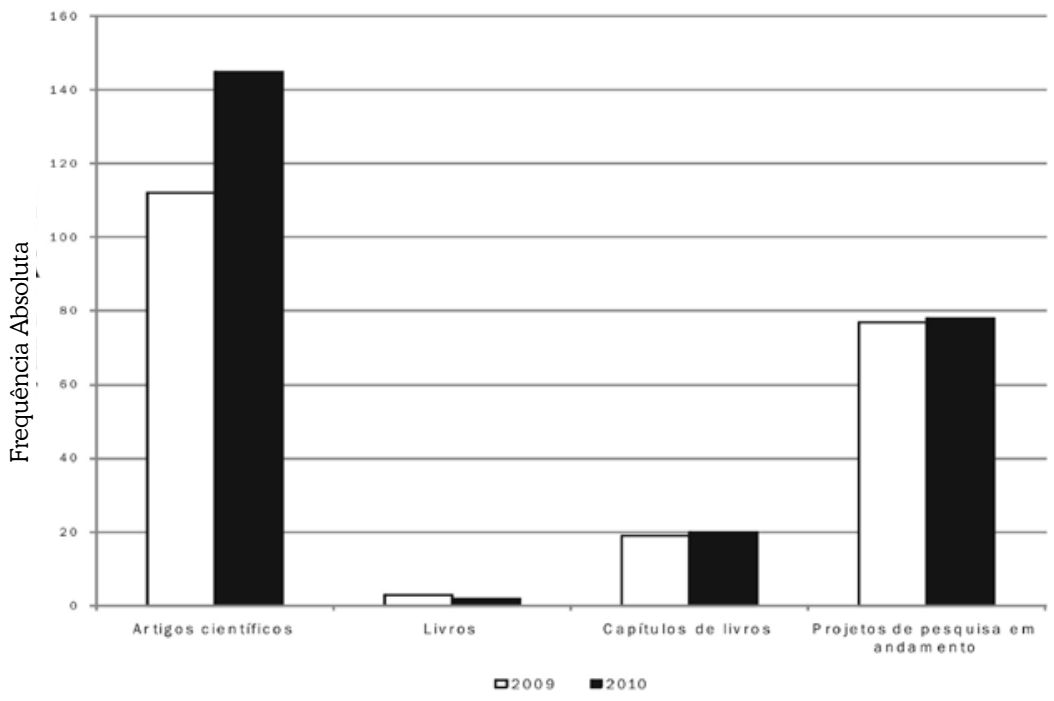

Fonte: Elaboração própria. 
No eixo de formação de pessoal, os indicadores relacionados às atividades de orientações de alunos, realizadas pelos pesquisadores do Citecs, mostraram que, no ano de 2009 e 2010, estavam sob orientação, respectivamente, um aluno de pós-doutorado (nos dois anos), 37 e 45 de doutorado, 36 e 35 de mestrado, sete e 14 de especialização e 25 e 51 de graduação. O indicador relacionado à oferta de cursos mostrou que o Citecs realizou 16 cursos em 2009 e 22 em 2010 (Figura 4).

Figura 4 - Distribuição dos indicadores de formação de pessoal, CITECS, 2009 e 2010.

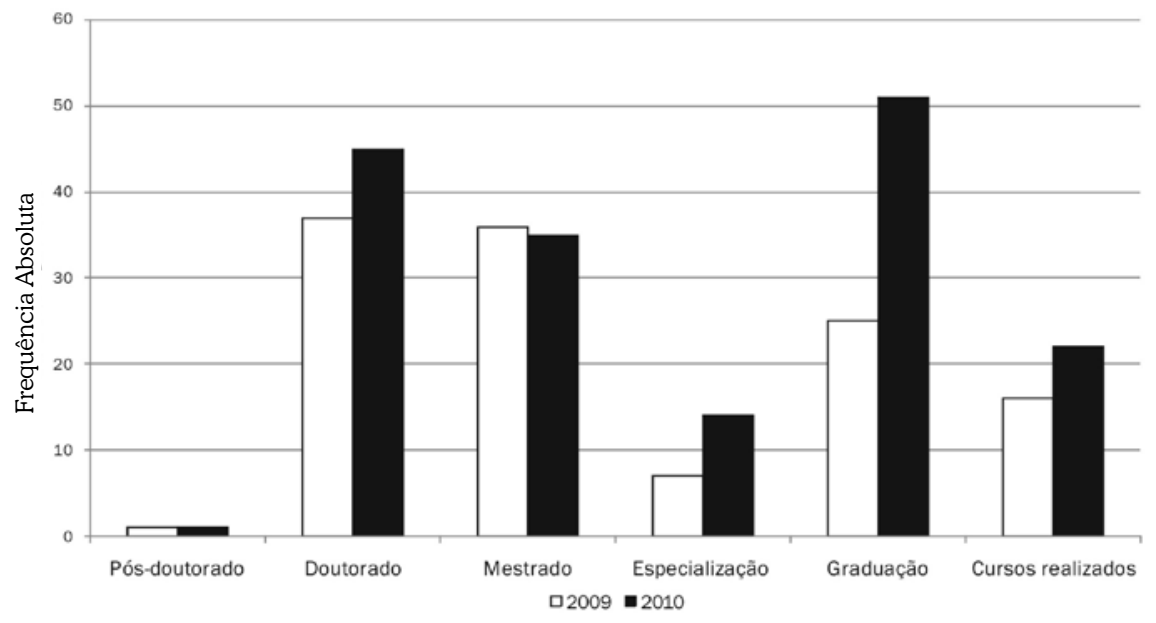

Fonte: Elaboração própria.

\section{Discussão}

As informações produzidas pelo presente estudo demonstram que, entre 2009 e 2010, houve ampliação das atividades realizadas nos quatro eixos. Esses resultados revelam que o Citecs tem atendido aos objetivos propostos pelo Programa dos INCT, realizando atividades de transferência do conhecimento e formação de pessoal, junto com a pesquisa. Quatro indicadores destacam-se por terem apresentado aumento entre os anos avaliados:

a) apoio à formulação de políticas na área da saúde;

b) atividades de educação científica;

c) inclusão de alunos de graduação em estágios de iniciação científica;

d) publicações científicas em conjunto.

O aumento do número de publicações científicas foi acompanhado pelo aumento do número de publicações científicas em conjunto. Estas últimas representavam, em 2009, 11\% do total de publicações e passaram para $22 \%$ em 2010. $\mathrm{O}$ aumento deste indicador sugere a existência de maior colaboração entre os 
grupos de pesquisa, após a constituição da rede e permite a avaliação da dinâmica e dos mecanismos estruturais de um sistema organizado em rede (BARABÁSI et al., 2002). Através desse indicador, identificam-se os pesquisadores com menos ou mais artigos em cooperação.

Além da rede de coautoria, neste estudo, são identificadas colaborações potenciais a serem desenvolvidas neste INCT, a partir de informações sobre áreas de atuação e de interesse comuns dos pesquisadores. A avaliação do eixo 1 mostrou, ainda, redução do intercâmbio de pesquisadores, atividade que poderia ampliar os vínculos com pesquisadores externos, expandindo o alcance da rede.

No eixo de transferência do conhecimento há melhorias em todos os indicadores, com exceção da formalização de novas parcerias com governos e empresas que permaneceu estável. Destacam-se as atividades de apoio à formulação de políticas e à elaboração de materiais técnicos para governos que se ampliaram em $100 \%$. O crescimento dessas atividades indica a existência de colaboração entre pesquisadores e gestores para transferência do conhecimento científico. A geração de conhecimento que possa ter impacto no sistema de saúde em um cenário marcado por restrição de recursos é de extrema importância (LOMAS, 1997). O Citecs tem atuado junto à gestão da saúde também através da participação em comitês técnicos de PEBD de governos e organizações internacionais, a exemplo da Organização Mundial da Saúde.

As atividades de educação científica também merecem ser destacadas. Em 2009, nenhuma atividade dessa natureza foi realizada, enquanto ocorreram sete atividades em 2010. Esse resultado representa um avanço, tendo em vista a importância da educação científica para o desenvolvimento social, ao preparar o cidadão para assimilar conhecimentos e informações de qualidade, aprimorando a sua capacidade de juízo crítico. O conhecimento científico é um importante capital do mundo civilizado, enquanto o analfabetismo científico representa um fator de aumento das desigualdades sociais (GAVIDIA, 1998).

Entre 2009 e 2010, houve crescimento de 14\% nas atividades de divulgação da ciência pelos meios de comunicação de massa. Assim como a educação científica, as atividades de divulgação dos resultados das pesquisas são ações que podem contribuir para o desenvolvimento de uma nação, na medida em que proporcionam a compreensão pública da ciência, propiciam aos indivíduos maior grau de autonomia, sendo, portanto, instrumentos para promover a democracia (BUENO, 1985; ALBAGLI, 1996).

No que diz respeito a ações de inovação, contrário aos demais indicadores, não houve avanços, durante os dois anos avaliados, visto que este INCT não registrou nenhuma patente. As patentes constituem um dos indicadores mais importantes da inovação e do desenvolvimento científico-tecnológico de um país (CHAIMOVICH, 2000). 
Apesar do esforço realizado pelo Ministério de Ciência e Tecnologia de incentivo ao registro de patentes, no Brasil, tem sido observado, não apenas neste INCT, como também em outros institutos de pesquisa, que o crescimento do número de publicações científicas não tem sido acompanhado do aumento da quantidade de patentes. Um dos caminhos apontados por documentos técnicos sobre Ciência, Tecnologia e Inovação para tentar superar este obstáculo é a realização de projetos em parceria universidade-empresa (BRASIL, 2010). Vale ressaltar que o tempo de existência do Citecs, de pouco mais de um ano, talvez não tenha sido suficiente para que surgissem os resultados nas atividades de inovação.

Observa-se ainda no eixo formação de pessoal uma ampliação no número de doutores (22\%) e de especialistas lato sensu (100\%) formados, enquanto o número de mestres se reduziu em 3\%. A orientação de estudantes de graduação obteve significativo aumento (104\%), demonstrando a existência de atividades voltadas para a formação de futuros pesquisadores. A participação em projetos de pesquisa desde a graduação permite que os alunos tenham acesso a um arcabouço teórico, a instrumentos metodológicos, além de criar condições para o despertar da vocação científica e para a descoberta de talentos potenciais (BASTOS et al, 2010).

Em consonância com esse tipo de ação, a inserção dos estudantes de graduação no campo de pesquisa e inovação tem sido amplamente estimulada pela Política de Ciência e Tecnologia no Brasil, através da criação do programa Ciência sem Fronteiras que "busca promover a consolidação, expansão e internacionalização da ciência e tecnologia, da inovação e da competitividade brasileira por meio do intercâmbio e da mobilidade internacional" (BRASIL, 2012).

\section{Considerações Finais}

O Instituto de Ciência, Inovação e Tecnologia em Saúde ampliou as ações nos quatro eixos avaliados, sendo que o eixo pesquisa apresentou maior avanço. A rede Citecs mostra-se coesa, principalmente através da colaboração em publicações científicas e projetos de pesquisa em andamento. Houve aumento em todos os indicadores de transferência de conhecimento, exceto a formalização de parcerias com governos e empresas. No eixo formação de pessoal, o destaque foi a inclusão de alunos de graduação e formação de especialistas lato sensu. O pouco tempo de existência do Instituto, menos de dois anos, pode explicar a inexistência de registro de patentes.

A partir dessa análise, constata-se que a matriz elaborada foi adequada para a avaliação do desempenho dessa rede de pesquisa, pois permitiu mensurar as ações desenvolvidas, considerando as metas desse INCT e apontar os pontos fortes e fracos dentro de cada eixo. Entretanto, o fato de ter sido aplicada em dois momentos distintos, somente a um único INCT, restringe a avaliação da robustez e acurácia da matriz. 
Dentre as contribuições desta, ressalta-se a possibilidade de identificar, não apenas as colaborações existentes entre os pesquisadores da rede, mas também as conexões potenciais. Além disso, a constituição da matriz em quatro eixos permitiu avaliar, conjuntamente, os indicadores de consolidação da rede e transferência de conhecimento, com os tradicionais indicadores de pesquisa e de formação de pessoal, o que representa um avanço em relação aos métodos bibliométricos existentes. Sugere-se que o método utilizado nesta avaliação pode ser adaptado para avaliar outras redes de pesquisa com objetivos similares.

\section{Referências}

ALBAGLI, S. Divulgação científica: informação cientifica para a cidadania? Ciência da informação, Brasília, v.25, p. 396-404, 1996.

ALMEIDA-FILHO, N. Sobre Redes. In: TEIXEIRA, F. (Org.). Gestão de Redes de Cooperação Interempresariais: em busca de novos espaços de aprendizado e inovação. Salvador: Casa da Qualidade, 2005.p. 5-9.

BARABÁSI, A. et al. Evolution of the Social Network of Scientific Collaborations. Physica A, v.311. p. 590-614, 2002.

BASTOS, F. et al. A Importância da Iniciação Científica para os Alunos de Graduação em Biomedicina. Revista Eletrônica Novo Enfoque, v.11, n.11, p.61- 66, 2010.

CONFERÊNCIA NACIONAL DE CIÊNCIA, TECNOLOGIA E INOVAÇÃO PARA O DESENVOLVIMENTO SUSTENTÁVEL, 4., 2010. In: Parcerias Estratégicas, v.15, n.31, 308 p., 2010.

BRASIL. Conselho Nacional de Desenvolvimento Científico e Tecnológico. Programa Ciência Sem Fronteiras. 2012. Disponível em: < http://www.cienciasemfronteiras.gov.br/web/csf> . Acesso em: 05 jan. 2012.

BRASIL. Ministério da Ciência e Tecnologia. Programa Institutos Nacionais de CET.2008. Disponível em:<www.cnpq.br/editais/ct/2008/docs/015_anexo.pdf>. Acesso em: 05 jan. 2012.

. PACTI 2007-2010: Principais Resultados e Avanços.2009. Disponível em: <http:// www.mct.gov.br/upd_blob/0211/211012.pdf>.Acesso em: 12 nov. 2011.

BUENO, W. C. Jornalismo Científico: Conceito e Unção. Ciência e Cultura, São Paulo, v. 37, n. 9, p. 1420-1427, 1985.

CHAIMOVICH, H. Brasil, Ciência, Tecnologia: Alguns Dilemas e Desafios. Estudos Avançados, São Paulo, v. 14, n. 40, Sept./Dec.2000.

DEAN, A. G. et al. Epi Info, Version 6.03: A Word Processing Database and Statistics Program for Public Health on IBM-Compatible Microcomputers. [Computer program]. Atlanta: Centers of Disease Control and Prevention, 1998.

GAVIDIA, V. Salud, Educación y Calidad de Vida: De Cómo Las Concepciones Del Profesorado Inciden en La Salud. Santa Fe de Bogotá: Magisterio, 1998.

GRIFFITHS, F. et al. The productivity of primary care research networks. British Journal of General Practice, v.50, p. 913-915, Nov. 2000. 
LOMAS, J. Improving Research Dissemination and Uptake in the Health Sector: Beyond the Sound of One Hand Clapping. Policy Commentary. Ontario: McMaster University Centre for Health Economics and Policy Analysis, 1997.

MORAES, M. A Ciência como Rede de Atores: Ressonâncias Filosóficas. Hist. Cienc. Saude, Manguinhos, v.11, n.2, p.321-333, 2004.

POLDONY, J. M.; PAGE, K. L. Network Forms of Organization. Annual Review of Sociology, v. 24, p.57-76, 1998.

Statistical Package for the Social Sciences forWindows (SPSS). Version 13.0. [Computer program]. Chicago: SPSS Inc., 2006.

UNITED KINGDOM. Office of Science and Technology. Disponível em http://www.ost.gov.uk. Acesso em 20/07/2011.

WEGNER, D.; RIBEIRO, J. Avaliação de Desempenho de Redes Horizontais de Empresas: Um Estudo Exploratório. Revista Alcance - Eletrônica, v. 18, n. 1, p. 59-74, 2011.

Recebido em: 16/01/2012.

Aceito em: 16/03/2012. 